\title{
NUMERICAL DISCREPANCIES OF USING A NONCONSERVATIVE FORMULATION OF THE COMPRESSIBLE GAS FLOW MODEL
}

\author{
Ferdinand E. Uilhoorn \\ Warsaw University of Technology, Gas Engineering Group \\ Warsaw, Poland \\ ferdinand.uilhoorn@pw.edu.pl
}

Received: 16 July 2019; Accepted: 15 January 2020

\begin{abstract}
In this article, we investigated the shock phenomenon in gas pipeline systems. We particularly address the numerical discrepancies introduced when using a primitive variable-based formulation of the compressible gas flow model. For the analysis, we compared two different schemes, namely, van Leer's second-order Monotonic Upstream-centered Scheme for Conservation Laws scheme (TVD-MUSCL) together with Roe's superbee slope limiter and the fifth-order accurate finite volume weighted essentially non-oscillatory scheme (WENO5-Z). For the numerical flux, we implemented the Rusanov solver. The time stepping was done with a strong stability preserving Runge-Kutta method. The method of manufactured solutions was used to verify the code accuracy. Based on a series of numerical experiments, we showed that the local errors become more visible if we use the WENO5-Z reconstruction.
\end{abstract}

MSC 2010: 74H15, $76 N 15$

Keywords: shock phenomenon, TVD-MUSCL, WENO-Z, numerical error

\section{Introduction}

Theoretical and computational fluid dynamics in pipeline systems have a very broad interest because of their practical applications. Several examples are oil and gas pipelines, piping systems under pressure in hydroelectric power stations and urban water and heating networks. In such systems, it is of utmost importance to understand the dynamics in the presence of shocks because it enables us to prevent or mitigate excessive high or low pressures in the system. This phenomenon, also known as the fluid hammer is often a result of rapid valve closure.

Most numerical models that simulate shock behavior in gas pipelines as a result of rapid valve closure use a primitive variable-based formulation [1-7]. ${ }^{1}$ The nonlinear hyperbolic partial differential equations (PDEs) describing fluid hammer for compressible and incompressible fluids are mostly written in terms of primitive variables, such as flow rate, or velocity and pressure because these variables are typically

$\overline{{ }^{1} \text { Although beyond the scope of this work, }}$ it also concerns water hammer simulations. 
measured. From a physical point of view, these variables are not conserved, therefore the system is still nonconservative. In the present study, we consider the well-known and frequently used isothermal gas flow model that is formulated as follows:

$$
\begin{gathered}
\frac{\partial p}{\partial t}+\frac{c^{2}}{A} \frac{\partial \dot{m}}{\partial x}=0 \\
\frac{\partial \dot{m}}{\partial t}+\frac{\partial}{\partial x}\left(\frac{\dot{m}^{2} c^{2}}{p A}\right)+A \frac{\partial p}{\partial x}+\frac{f_{r} c^{2}}{2 d A} \frac{\dot{m}^{2}}{p}=0,
\end{gathered}
$$

for $x \in \mathbb{R}, t>0$ with pressure $p$, speed of sound $c$, cross-sectional area $A$, mass flow rate $\dot{m}$, diameter $d$ and friction factor $f_{r}$. This set of equations in a nonconservative form with or without the convective inertia term is frequently used in engineering practice. For more accurate predictions, the energy equation is included $[5,8]$ but even then we still have a nonconservative form.

In [9-12], it was shown that such primitive variable-based expressions are valid if we have smooth solutions but not in the presence of shocks because nonconservative formulations converge to wrong weak solutions. This was demonstrated by imposing discontinuities in the initial conditions. In this work, we examine a situation in which we have discontinuities in the boundary conditions at the outlet node. This appears when we are simulating hydraulic shocks caused by rapid valve closure, for example. Thus we solve Eqs. (1) and (2) and their equivalent form, namely the one that is physically conserved and we compare the results.

The finite volume method, is in general, the method of choice to handle shock waves described by hyperbolic PDEs because of the shock-capturing properties. It is characterized by its accuracy, efficiency and robustness when solving the conservation laws. For both formulations, we examine two reconstruction schemes. First, we apply van Leer's second-order TVD-MUSCL (Monotonic Upstream-centered Scheme for Conservation Laws) scheme. Here spurious oscillations are avoided by using a total variation diminishing (TVD) limiter. This scheme is second-order accurate if we have smooth solutions but degenerates to first order at shocks. The fifth-order accurate finite-volume weighted essentially non-oscillatory (WENO) scheme [13] is the second scheme we implement. In particular WENO-Z [14], which shows less dissipation and higher resolution compared to the classical WENO reconstruction. It has fifth-order convergence not only in smooth regions but also at critical points. The reason for using these schemes is, that they satisfactorily handled shocks and sharp interfaces. For the numerical flux, we use the Rusanov Riemann solver $[15,16]$. The time integration is done with the three-stage third-order strong stability preserving (SSP) Runge-Kutta (RK) scheme [17]. Numerical experiments are done for a pipeline section with a shut-off valve at the outlet node. 


\section{Mathematical model}

The compressible flow equations (1) and (2) in vector form can be written as follows:

$$
u_{t}+f(u)_{x}=s(u),
$$

with

$$
u=\left(\begin{array}{c}
p \\
\dot{m}
\end{array}\right), \quad f(u)=\left(\begin{array}{c}
\frac{c^{2}}{A} \dot{m} \\
\dot{m}^{2} c^{2} \\
p A
\end{array}\right), \quad s(u)=\left(\begin{array}{c}
0 \\
-\frac{f_{r} c^{2}}{2 d A} \frac{\dot{m}^{2}}{p}
\end{array}\right),
$$

where $f(u)$ and $s(u)$ are the flux and source term, respectively. According to Toro [12], such formulation is conservative in a mathematical sense, but not from a physical perspective. The conservation principle for pressure does not make sense, physically. To make the set of equations physically conserved, we use the ideal equation of state $p / \rho=c^{2}$ with $\rho$ denoted as the density. This closure relation enables us to write the system as follows:

$$
w_{t}+F(w)_{x}=\mathscr{S}(w),
$$

with

$$
w=\left(\begin{array}{c}
\rho \\
\rho v
\end{array}\right), \quad F(w)=\left(\begin{array}{c}
\rho v \\
\frac{(\rho v)^{2}}{\rho}+c^{2} \rho
\end{array}\right), \quad \mathscr{S}(w)=\left(\begin{array}{c}
0 \\
-\frac{f_{r}}{2 d} \frac{(\rho v)^{2}}{\rho}
\end{array}\right),
$$

whereas the state variables are conserved. The Jacobian matrix $A(w)=\partial F(w) / \partial w$ is defined as

$$
A(w)=\left[\begin{array}{cc}
0 & 1 \\
c^{2}-v^{2} & 2 v
\end{array}\right],
$$

where $v$ is the velocity. The eigenvalues of matrix $A$ are $\lambda_{1}=v-c$ and $\lambda_{2}=v+c$ with corresponding right eigenvectors

$$
R=\left[\begin{array}{cc}
1 & 1 \\
v-c & v+c
\end{array}\right]
$$

The system is hyperbolic because all eigenvalues of the flux Jacobian matrix are real and the set of corresponding eigenvectors is complete. The same results are obtained for the nonconservative formulation (3). 


\section{Numerical method}

We start to discretize the governing equations on the domain $\mathscr{D}=\{x \in[0, L]\}$ into $N-1$ equal spaced cells, i.e., $\Delta x=L /(N-1)$ where $L$ is the pipe length. For each cell, we define midpoint $x_{i}$ and its edges $x_{i-\frac{1}{2}}$ and $x_{i+\frac{1}{2}}$. The semi-discretized form of flow model (4) but similar for Eq. (3) can be written as

$$
\frac{\mathrm{d}}{\mathrm{d} t} w_{i}=-\frac{1}{\Delta x}\left(F_{i+\frac{1}{2}}-F_{i-\frac{1}{2}}\right)+\mathscr{S}_{i},
$$

where $w_{i}$ approximates the cell average of the dependent variables. The numerical flux $F_{i \pm \frac{1}{2}}$ is defined at the left and right cell boundaries and $\mathscr{S}_{i}$ is the source term. Equation (7) can be transformed in the form

$$
\frac{\mathrm{d} w}{\mathrm{~d} t}=\mathscr{F}(w), \quad t \in\left[0, t_{f}\right],
$$

where the nonlinear operator $\mathscr{F}(w)$ reads

$$
\mathscr{F}(w)=-\frac{1}{\Delta x}\left(F_{i+\frac{1}{2}}-F_{i-\frac{1}{2}}\right)+\mathscr{S}_{i}
$$

\subsection{Temporal discretization}

Assuming that the approximation at time instant $n$ is known, then using the $\operatorname{SSPRK}(3,3)$ scheme we obtain the solution at $n+1$ as follows:

$$
\begin{aligned}
w_{i}^{(1)} & =w_{i}^{n}+\Delta t \mathscr{F}\left(w_{i}^{n}\right), \\
w_{i}^{(2)} & =\frac{3}{4} w_{i}^{n}+\frac{1}{4} w_{i}^{(1)}+\frac{1}{4} \Delta t \mathscr{F}\left(w_{i}^{(1)}\right), \\
w_{i}^{(n+1)} & =\frac{1}{4} w_{i}^{n}+\frac{2}{3} w_{i}^{(2)}+\frac{2}{3} \Delta t \mathscr{F}\left(w_{i}^{(2)}\right) .
\end{aligned}
$$

For the numerical stability, the time step depends on the Courant number:

$$
C=\max (|\lambda|) \frac{\Delta t}{\Delta x}
$$

with $C \leqslant 1$ and $\lambda$ is the maximum value over all eigenvalues in all cells.

\subsection{MUSCL-TVD scheme}

The MUSCL-TVD reconstruction of [18] assumes a piecewise-linear interpolation from the average values. The reconstruction for each component is defined by

$$
w_{i+1 / 2}^{L}=w_{i}+\frac{1}{2} \phi\left(\theta_{i}\right)\left(w_{i+1}-w_{i}\right),
$$


and

$$
w_{i+1 / 2}^{R}=w_{i+1}-\frac{1}{2} \phi\left(\theta_{i}\right)\left(w_{i+2}-w_{i+1}\right)
$$

where $\theta$ is

$$
\theta_{i}=\frac{w_{i}-w_{i-1}}{w_{i+1}-w_{i}}
$$

and $\phi$ is a slope limiter function [19] that ensures TVD, in the sense that it avoids spurious oscillations around discontinuities. The anti-diffusion flux is determined by $\phi(\theta)$ and when its value increases less numerical dissipation is observed but more dissipation is expected when it decreases. In the former situation, the resolution is higher at the discontinuities or shocks. This scheme is second-order accurate for smooth parts of the domain but reduces to a first order at local smooth extrema [20]. Examples of most usual limiter functions are

(i) van Leer limiter: $\phi(\theta)=\frac{\theta+|\theta|}{1+|\theta|}$,

(ii) monotonized central (MC) limiter:

$$
\phi(\theta)=\max (0, \min (2 \theta,(1+\theta) / 2,2)),
$$

(iii) van Albada limiter: $\phi(\theta)=\frac{\theta^{2}+\theta}{\theta^{2}+1}$,

(iv) minmod limiter: $\phi(\theta)=\max (0, \min (1, \theta))$,

(v) superbee limiter:

$$
\phi(\theta)=\max (0, \min (2 \theta, 1), \min (\theta, 2)) .
$$

In this work, we used Roe's superbee flux limiter because it is considered as a good compromise between accuracy and computational efficiency [21].

\subsection{WENO5-Z scheme}

The classical fifth-order WENO reconstruction developed by [13] can be written as

$$
\begin{aligned}
w_{i+\frac{1}{2}}^{L} & =\omega_{0}\left(\frac{1}{3} w_{i-2}-\frac{7}{6} w_{i-1}+\frac{11}{6} w_{i}\right)+\omega_{1}\left(-\frac{1}{6} w_{i-1}+\frac{5}{6} w_{i}+\frac{1}{3} w_{i+1}\right) \\
& +\omega_{2}\left(\frac{1}{3} w_{i}+\frac{5}{6} w_{i+1}-\frac{1}{6} w_{i+2}\right)
\end{aligned}
$$

and

$$
\begin{aligned}
w_{i-\frac{1}{2}}^{R} & =\omega_{0}\left(\frac{1}{3} w_{i}+\frac{5}{6} w_{i-1}-\frac{1}{6} w_{i-2}\right)+\omega_{1}\left(-\frac{1}{6} w_{i+1}+\frac{5}{6} w_{i}+\frac{1}{3} w_{i-1}\right) \\
& +\omega_{2}\left(\frac{1}{3} w_{i+2}-\frac{7}{6} w_{i+1}+\frac{11}{6} w_{i}\right)
\end{aligned}
$$


respectively. The nonlinear weights are defined as

$$
w_{r}=\frac{\alpha_{r}}{\alpha_{0}+\alpha_{1}+\alpha_{2}}, \quad \alpha_{r}=\frac{d_{r}}{\left(\varepsilon+\beta_{r}\right)^{2}}, \quad r=0,1,2,
$$

with the following smoothness indicators

$$
\begin{aligned}
& \beta_{0}=\frac{13}{12}\left(w_{i}-2 w_{i+1}+w_{i+2}\right)^{2}+\frac{1}{4}\left(3 w_{i}-4 w_{i+1}+w_{i+2}\right)^{2}, \\
& \beta_{1}=\frac{13}{12}\left(w_{i-1}-2 w_{i}+w_{i+1}\right)^{2}+\frac{1}{4}\left(w_{i-1}-w_{i+1}\right)^{2}, \\
& \beta_{2}=\frac{13}{12}\left(w_{i-2}-2 w_{i-1}+w_{i}\right)^{2}+\frac{1}{4}\left(w_{i-2}-4 w_{i-1}+3 w_{i}\right)^{2} .
\end{aligned}
$$

By symmetry, the optimal linear weights for $w_{i-\frac{1}{2}}^{R}$ at $x_{i-\frac{1}{2}}$ are $d_{0}=3 / 10, d_{1}=3 / 5$ and $d_{2}=1 / 10$ and for $w_{i+\frac{1}{2}}^{L}$ at $x_{i+\frac{1}{2}}$ we define $d_{0}=1 / 10, d_{1}=3 / 5$ and $d_{2}=3 / 10$. To avoid division by zero, we set $\varepsilon=10^{-6}$.

Here, the choice of the nonlinear weights and smoothness indicators in the classical WENO scheme are not unique and can be modified to improve the accuracy at the critical points. In [22] it was shown that for the classical fifth-order WENO scheme [13], the nonlinear weights do not satisfy the conditions for fifth-order convergence. To overcome this shortcoming, a mapping function was introduced leading to the so-called WENO-M scheme but at the cost of computation time. Therefore, Borges et al. [14] developed a more efficient scheme, denoted as WENO-Z, which contains different smoothness indicators. This scheme improved the convergence properties and reduced the numerical dissipation at shocks. In this reconstruction, the nonlinear weights are defined as follows:

$$
w_{r}^{z}=\frac{\alpha_{r}^{z}}{\sum_{l=0}^{2} \alpha_{l}^{z}}, \quad \alpha_{r}^{z}=d_{r}\left(1+\left(\frac{\tau_{5}}{\beta_{r}+\varepsilon}\right)^{q}\right),
$$

with $\tau_{5}=\left|\beta_{0}-\beta_{2}\right|$ and $r=\{0,1,2\}, q=1,2, \ldots$. Fourth order accuracy is achieved for $q=1$ while if $q=2$ fifth order accuracy is ensured at the critical points.

\subsection{Rusanov flux}

The left and right states are constructed by the schemes at the cell edges. In this work, we use the Rusanov solver $[15,16]$ to calculate the corresponding fluxes at these cell boundaries. This solver is defined as follows:

$$
F_{i+\frac{1}{2}}=\frac{1}{2}\left(F^{R}+F^{L}\right)-\frac{\psi_{i+\frac{1}{2}}}{2}\left(w_{i+\frac{1}{2}}^{R}-w_{i+\frac{1}{2}}^{L}\right),
$$


where $\psi_{i+\frac{1}{2}}$ is the local wave speed between cells $i$ and $i+1$. Here $F^{R}=F\left(w_{i+\frac{1}{2}}^{R}\right)$ and $F^{L}=F\left(w_{i+\frac{1}{2}}^{L}\right)$ refer to flux using the right and left reconstructed state, respectively.

\section{Code verification}

In this section, we carry out a convergence study to verify whether the schemes are implemented correctly. We use the method of manufactured solutions because it utilizes exact solutions and is therefore considered as most rigorous. First, a priori analytic physically solutions are defined. We use the following functions

$$
\begin{gathered}
\rho(x, t)=\rho_{0}+\alpha_{0} \sin \left(\frac{2 \pi x}{L}\right) \cos \left(\frac{2 \pi t}{T_{0}}\right), \\
\rho v(x, t)=\rho v_{0}+\frac{\alpha_{0} L}{T_{0}} \cos \left(\frac{2 \pi x}{L}\right) \sin \left(\frac{2 \pi t}{T_{0}}\right) .
\end{gathered}
$$

The manufactured solutions are substituted into model (3). This yields into a modified system that enables us to determine the analytical solution of the source term denoted here as $h(x, t)$. For more details about this procedure, the reader is referred to Roy [23]. Hence, we write

$$
u_{t}+f(u)_{x}-s(u)=h(x, t) .
$$

The governing equations are then discretized and solved on different meshes. The discretization error was calculated using the $L_{2}$-norm of the error whereas the exact solutions are obtained from the manufactured solutions. The results are shown in Figure 1 for both schemes using the conservative formulation. It shows that the order of accuracy decays according to the theoretical ones. For the TVD-MUSCL and WENO5-Z reconstructions, we computed a slope of 1.98 and 5.04, respectively.

\section{Numerical results}

In this section, we conduct a series of numerical experiments for a $20 \mathrm{~km}$ pipeline with a diameter of $0.5 \mathrm{~m}$. Other model parameters are $c=348.5 \mathrm{~m} \mathrm{~s}^{-1}$ and $f_{r}=0.008$. After $5 \mathrm{~min}$, the valve at the outlet node was instantaneously closed for $20 \mathrm{~min}$ and then opened again. The mass flow rate increased from zero back to $70 \mathrm{~kg} \mathrm{~s}^{-1}$ (see Figure 2). The pressure in the pipeline is maintained at 5.0 MPa. The initial conditions are defined as

$$
\frac{\partial \dot{m}}{\partial x}(x, 0)=0, \quad \frac{\partial p}{\partial x}(x, 0)=-\frac{f_{r} c^{2} \dot{m}^{2} p}{2 d\left(A^{2} p^{2}-c^{2} \dot{m}^{2}\right)},
$$


and the boundary conditions can be written as

$$
p(0, t)=p_{0}(t), \quad \dot{m}(L, t)=\dot{m}_{L}(t),
$$

where $p_{0}(t)=5.0 \mathrm{MPa}$ and

$$
\dot{m}_{L}(t)= \begin{cases}\dot{m}_{0}, & \text { if } t / \mathrm{s} \leqslant 300 \mathrm{~s} \\ 0, & \text { if } 300<t / \mathrm{s} \leqslant 1500 \\ \dot{m}_{0}, & \text { otherwise }\end{cases}
$$

with $\dot{m}_{0}=70 \mathrm{~kg} \mathrm{~s}^{-1}$. Of course, one can argue if such an instantaneous valve closure is practically realistic. Moreover, different valve closure laws will show different results. Nevertheless, our main goal is to demonstrate the consequences of using a nonconservative expression of the governing equations while using two different types of reconstruction schemes with a different order of accuracy. The transformation to the conserved variables $\rho$ and $\rho v$ is done using the equation of state and cross-sectional area. For comparison, we need to use a fixed time step because minor differences might occur if the time step is based on the Courant number. The spatial-temporal evolution of pressure and mass flux is illustrated in Figure 3 and clearly shows the discontinuous character of the solutions. The gas adjacent to the valve was brought immediately to rest when the valve closed. This results in a backward pressure and flow rate wave. The reverse flow decreases the pressure and causes oscillations in pressure and flow.

To indicate the numerical consequences of using the nonconservative formulation, the maximum of the absolute and relative errors were calculated. This is done as follows

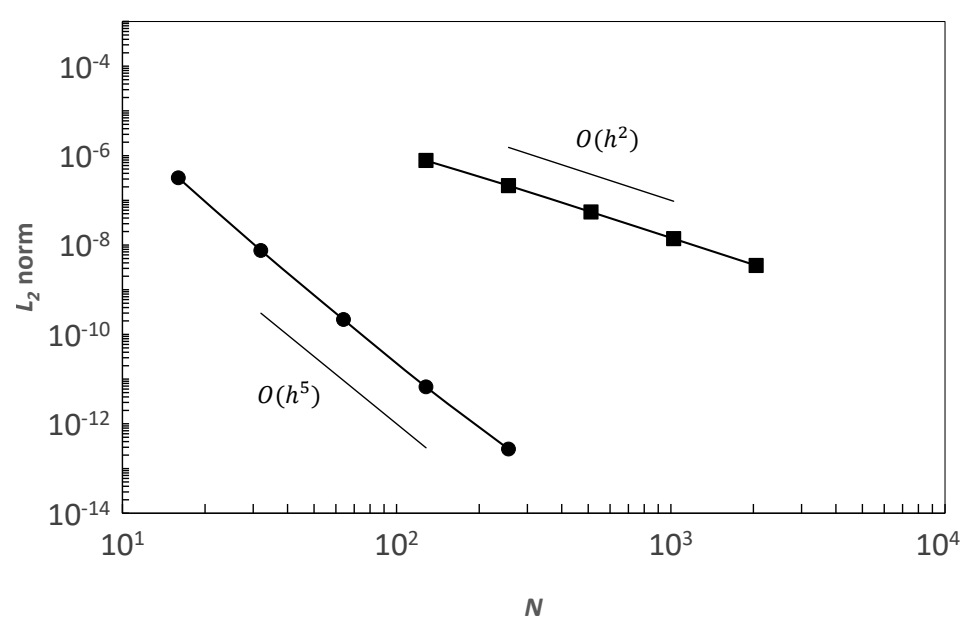

Fig. 1. Convergence for TVD-MUSCL and WENO5-Z reconstructions with parameters: $C=0.5$, $\alpha_{0}=10^{-4}, L=0.1, \rho_{0}=40$ and $\rho v_{0}=120$ and $T_{0}=0.1$. (-) TVD-MUSCL $(\bullet-)$ WENO5-Z 


$$
\begin{aligned}
& e_{\mathrm{abs}}=\max _{i j}\left(\left|y_{\mathrm{nc}, i j}-y_{\mathrm{c}, i j}\right|\right), \\
& e_{\mathrm{rel}}=\max _{i j}\left(\frac{\left|y_{\mathrm{nc}, i j}-y_{\mathrm{c}, i j}\right|}{\left|y_{\mathrm{c}, i j}\right|}\right),
\end{aligned}
$$

where $y_{\mathrm{nc}}$ and $y_{\mathrm{c}}$ represent the solutions of the nonconservative and conservative expressions, respectively. The solutions from the conservative expression are considered as a reference situation but are not exact. The maximum errors for different grid densities and time steps in the spatial and temporal domain are shown in Tables 1-4.

Based on the results, we conclude that if we use the TVD-MUSCL scheme the results for the conservative and nonconservative formulation are almost equivalent even

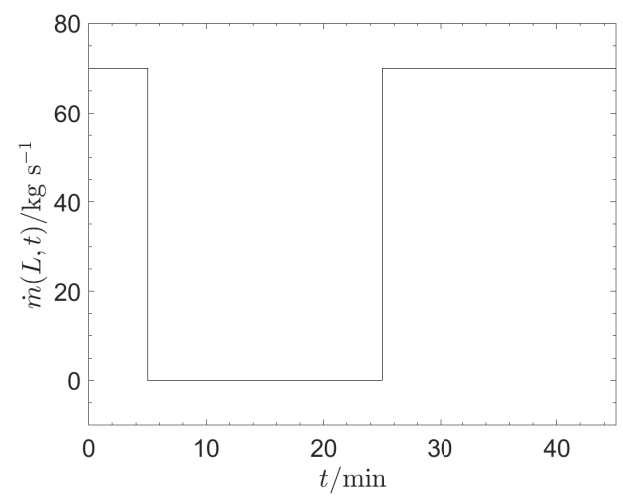

Fig. 2. Boundary condition for the mass flow rate at the valve

Table 1. Numerical discrepancies for different number of discretization points using MUSCL-TVD and $\Delta t=0.05 \mathrm{~s}$

\begin{tabular}{rcccc}
\hline$N$ & $e_{p} / \%$ & $e_{\dot{m}} / \%$ & $\max \left|e_{p}\right| / \mathrm{Pa}$ & $\max \left|e_{\dot{m}}\right| / \mathrm{kg} \mathrm{s}^{-1}$ \\
\hline 40 & $1.29 \times 10^{-12}$ & $9.37 \times 10^{-9}$ & $6.43 \times 10^{-8}$ & $5.14 \times 10^{-11}$ \\
80 & $2.82 \times 10^{-12}$ & $3.84 \times 10^{-10}$ & $1.41 \times 10^{-7}$ & $7.41 \times 10^{-11}$ \\
160 & $4.23 \times 10^{-11}$ & $2.55 \times 10^{-7}$ & $2.11 \times 10^{-6}$ & $1.48 \times 10^{-9}$ \\
320 & $1.01 \times 10^{-11}$ & $2.67 \times 10^{-10}$ & $4.73 \times 10^{-7}$ & $1.86 \times 10^{-10}$ \\
\hline
\end{tabular}

Table 2. Numerical discrepancies for different time steps using TVD-MUSCL and $N=320$

\begin{tabular}{ccccc}
\hline$\Delta t / \mathrm{s}$ & $e_{p} / \%$ & $e_{\dot{m}} / \%$ & $\max \left|e_{p}\right| / \mathrm{Pa}$ & $\max \left|e_{\dot{m}}\right| / \mathrm{kg} \mathrm{s}^{-1}$ \\
\hline 0.025 & $8.50 \times 10^{-11}$ & $3.97 \times 10^{-7}$ & $4.25 \times 10^{-6}$ & $2.28 \times 10^{-9}$ \\
0.050 & $1.01 \times 10^{-11}$ & $2.67 \times 10^{-10}$ & $4.73 \times 10^{-7}$ & $1.86 \times 10^{-10}$ \\
0.075 & $4.29 \times 10^{-12}$ & $1.71 \times 10^{-10}$ & $2.11 \times 10^{-7}$ & $1.20 \times 10^{-10}$ \\
0.1 & $1.89 \times 10^{-12}$ & $5.64 \times 10^{-11}$ & $9.41 \times 10^{-8}$ & $3.92 \times 10^{-11}$ \\
\hline
\end{tabular}



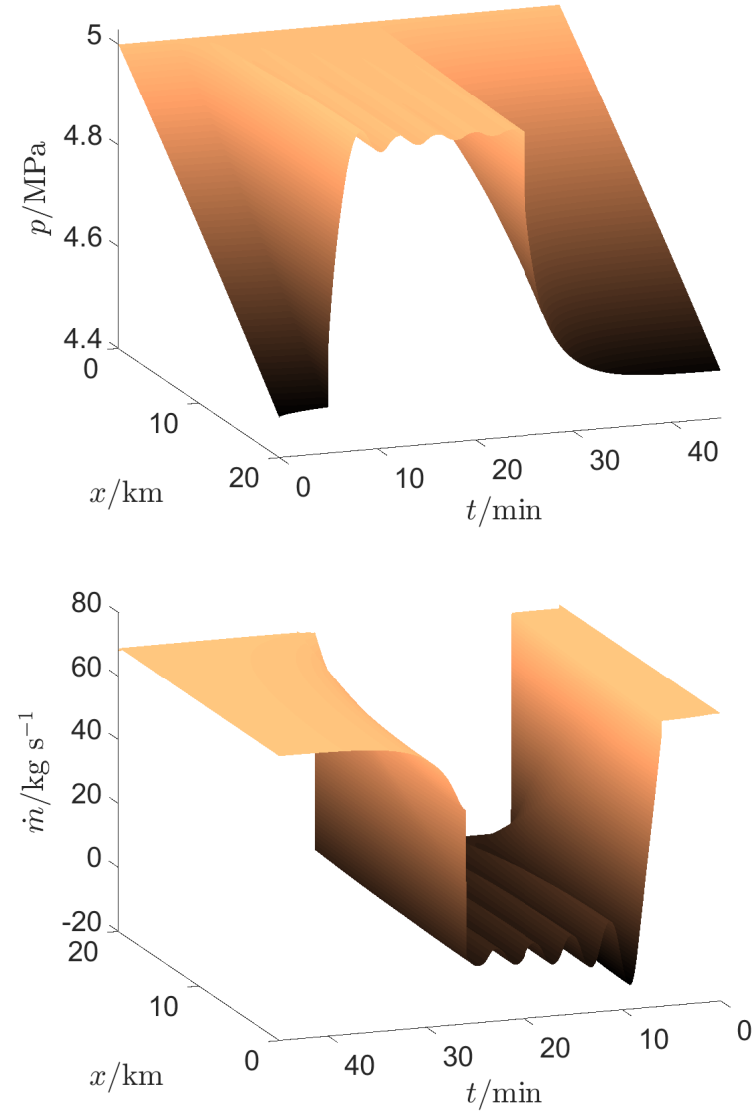

Fig. 3. Spatial-temporal evolution of pressure and mass flow rate

Table 3. Numerical discrepancies for different number of discretization points using WENO and $\Delta t=0.05 \mathrm{~s}$

\begin{tabular}{rcccc}
\hline$N$ & $e_{p} / \%$ & $e_{\dot{m}} / \%$ & $\max \left|e_{p}\right| / \mathrm{Pa}$ & $\max \left|e_{\dot{m}}\right| \mathrm{kg} \mathrm{s}^{-1}$ \\
\hline 40 & $5.46 \times 10^{-4}$ & 0.27 & 27.2 & $1.58 \times 10^{-2}$ \\
80 & $1.01 \times 10^{-3}$ & 1.08 & 50.5 & $2.44 \times 10^{-2}$ \\
160 & $1.32 \times 10^{-3}$ & 1.55 & 65.8 & $3.51 \times 10^{-2}$ \\
320 & $2.00 \times 10^{-3}$ & 0.07 & 95.0 & $3.92 \times 10^{-2}$ \\
\hline
\end{tabular}

Table 4. Numerical discrepancies for different time steps using WENO and $N=320$

\begin{tabular}{rcccc}
\hline$\Delta t / \mathrm{s}$ & $e_{p} / \%$ & $e_{\dot{m}} / \%$ & $\max \left|e_{p}\right| / \mathrm{Pa}$ & $\max \left|e_{\dot{m}}\right| / \mathrm{kg} \mathrm{s}^{-1}$ \\
\hline 0.025 & $1.89 \times 10^{-3}$ & $6.35 \times 10^{-2}$ & 89.4 & $3.69 \times 10^{-2}$ \\
0.050 & $2.00 \times 10^{-3}$ & $6.59 \times 10^{-2}$ & 95.0 & $3.92 \times 10^{-2}$ \\
0.075 & $1.57 \times 10^{-3}$ & 1.88 & 74.3 & $3.64 \times 10^{-2}$ \\
0.1 & $1.35 \times 10^{-3}$ & 2.07 & 67.3 & $4.34 \times 10^{-2}$ \\
\hline
\end{tabular}



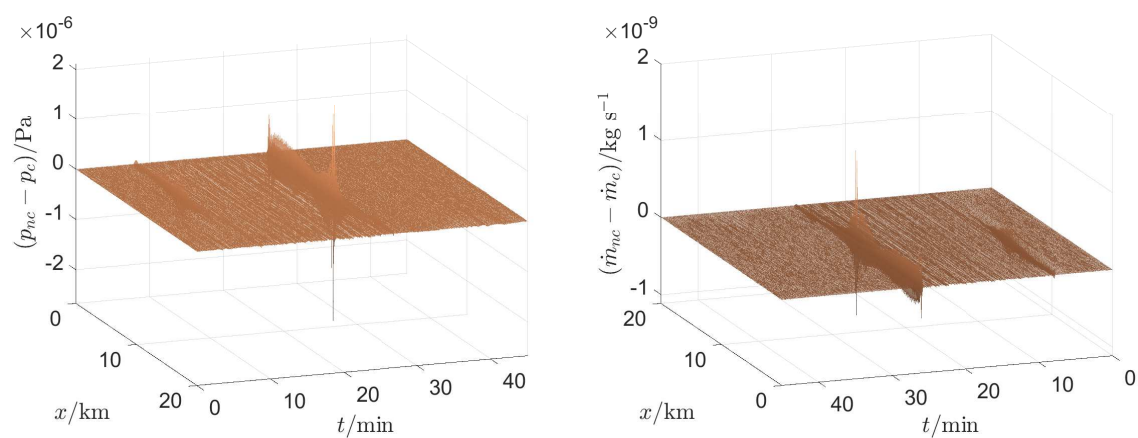

Fig. 4. Error evolution using TVD-MUSCL scheme with $N=160$ and $\Delta t=0.05 \mathrm{~s}$
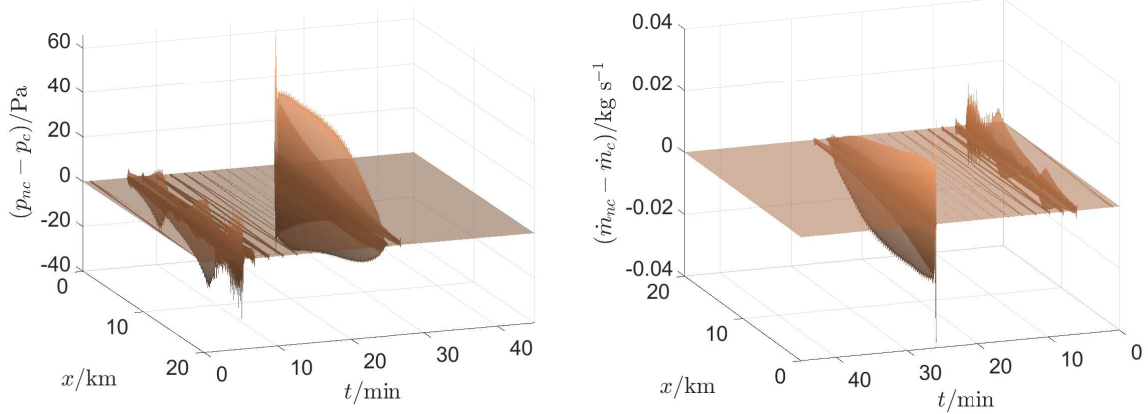

Fig. 5. Error evolution using WENO5-Z scheme with $N=160$ and $\Delta t=0.05 \mathrm{~s}$

at the discontinuities. The numerical discrepancies at each time step and grid point for this scheme are illustrated in Figure 4. Although the differences are not significant, we still see that higher values are observed at the discontinuities. This scheme is first order accurate at critical points. If we repeat the computations for WENO-Z, which is fifth order accurate, we observe more significant errors, especially for the mass flow rate (see Fig. 5). All maximum values were recorded near the moment of the valve opening or closing but at different grid nodes. A more dense grid causes an increase in pressure error. For the mass flow rate, we observe an increasing trend only up to and including $N=160$. If we double the number of grid points, we see a steep decline in error for the mass flow rate but the pressure error increases simultaneously. It should be noted that the maximum values with $N \leqslant 160$ were recorded in the neighborhood of valve opening ( $t \approx 25.9 \mathrm{~min}$ ). For $N=320$ the maximum errors were observed near valve closure $(t \approx 5.4 \mathrm{~min})$. If we set the number of grid points to $N=320$ and vary the time step, we see that a larger step increases the error for the mass flow rate while it decreases for the pressure. Depending on the time step, the maximum values were observed near the valve opening and closure. 


\section{Conclusions}

In this work, we adopted the compressible gas flow model that was approximated using the second-order TVD-MUSCL and fifth-order accurate WENO5-Z schemes. The Rusanov scheme was used to compute the fluxes at the cell edges. Two possible formulations of the gas flow model, namely the primitive variable-based and physical conserved one were investigated. Results showed that the numerical discrepancies between the two formulations is small if we use the classical TDV-MUSCL scheme. The differences become much more significant if we apply the fifth-order accurate WENO5-Z scheme. Hence, it seems that low order schemes are not able to detect the discrepancy between these two formulations. Although the primitive variable expression of the flow model is frequently used while simulating rapid valve closure, the physical conservative formulation is preferred. On the other hand, the local errors remain relatively small.

\section{References}

[1] Subani, N., \& Amin N. (2015). Analysis of water hammer with different closing valve laws on transient flow of hydrogen-natural gas mixture. Abstract and Applied Analysis, 510675, 1-12, DOI: $10.1155 / 2015 / 510675$.

[2] Uilhoorn, F.E. (2016). Estimating rapid flow transients using extended Kalman filter. Silesian Journal of Pure and Applied Mathematics, 6, 1, 97-110.

[3] Gyrya, V., \& Zlotnik, A. (2019). An explicit staggered-grid method for numerical simulation of large-scale natural gas pipeline networks. Applied Mathematical Modelling, 65, 34-51, DOI: 10.1016/j.apm.2018.07.051.

[4] Kiuchi, T. (1994). An implicit method for transient gas flows in pipe networks. International Journal of Heat and Fluid Flow, 15, 5, 378-383, DOI: https://doi.org/10.1016/0142-727X(94) 90051-5.

[5] Abbaspour, M., \& Chapman, K.S. (2008). Nonisothermal transient flow in natural gas pipeline. Journal of Applied Mechanics, 75, 3, 1-8, DOI:10.1115/1.2840046.

[6] Ding, Y., \& Li, Y. (2010). Study on effect of valve operation on transient flow in natural gas pipelines. International Conference on Digital Manufacturing \& Automation, Changsha, 463-466, DOI: 10.1109/ICDMA.2010.359.

[7] Greyvenstein, G.P. (2002). An implicit method for the analysis of transient flows in pipe networks. Numerical Methods in Engineering, 53, 5, 1127-1143, DOI: 10.1002/nme.323.

[8] Chaczykowski, M. (2010). Transient flow in natural gas pipeline - The effect of pipeline thermal model. Applied Mathematical Modelling, 34, 4, 1051-1067, DOI: 10.1016/j.apm.2009.07.017.

[9] Hou, T., \& Le Floch, P.G. (1994). Why nonconservative schemes converge to wrong solutions: error analysis. Mathematics of Computation, 62, 206, 497-530, DOI: 10.2307/2153520.

[10] Toro, E.F. (1994). Defects of Conservative Approaches and Adaptive Primitive-Conservative Schemes for Computing Solutions to Hyperbolic Conservation Laws. Technical report MMU 9401, Department of Mathematics and Physics, Manchester Metropolitan University, UK.

[11] Toro, E.F. (1995). On Adaptive Primitive-Conservative Schemes for Conservation Laws. M.M. Hafez (Ed.), Sixth International Symposium on Computational Fluid Dynamics, Vol. 3, LakeTahoe, Nevada, USA, 288-1293. 
[12] Toro, E.F. (2009). Riemann Solvers and Numerical Methods for Fluid Dynamics - A Practical Introduction. 3rd Edition, Berlin, Heidelberg: Springer-Verlag.

[13] Jiang, G.-S., \& Shu, C.-W. (1996). Efficient implementation of weighted ENO schemes. Journal of Computational Physics, 126, 1, 202-228, DOI:10.1006/jcph.1996.0130.

[14] Borges, R., Carmona, M., Costa, B., \& Don, W.S. (2008). An improved weighted essentially non-oscillatory scheme for hyperbolic conservation laws. Journal of Computational Physics, 227, 6, 3191-3211, DOI: 10.1016/j.jcp.2007.11.038.

[15] Rusanov, V. (1961). Calculation of intersection of non-steady shock waves with shock waves with obstacles. USSR Computational Mathematics and Mathematical Physics, 1, 267-279.

[16] Rusanov, V. (1962). The calculation of the interaction of non-stationary shock waves and obstacles. USSR Computational Mathematics and Mathematical Physics, 1, 2, 304-320.

[17] Shu, C.-W., \& Osher, S. (1988). Efficient implementation of essentially non-oscillatory shockcapturing schemes. Journal of Computational Physics, 77, 2, 439-471, DOI: 10.1016/00219991(88)90177-5.

[18] van Leer, B. (1979). Towards the ultimate conservative difference scheme V. A second-order sequel to Godunov method. Journal of Computational Physics, 32, 101-136, DOI: 10.1016/ 0021-9991(79)90145-1.

[19] Sweby, P.K. (1984). High resolution schemes using flux limiters for hyperbolic conservation laws. SIAM Journal of Numerical Analysis, 21, 5, 995-1011.

[20] Hesthaven, J.S. (2018). Numerical Methods for Conservation Laws: From Analysis to Algorithms: From Analysis to Algorithm, SIAM.

[21] Griffiths, G., \& Schiesser, W. (2011). Traveling Wave Analysis of Partial Differential Equations: Numerical and Analytical Methods with Matlab and Maple. Academic Press.

[22] Henrick, A.K., Aslam, T.D., \& Powers, J.M. (2005). Mapped weighted essentially non-oscillatory schemes: achieving optimal order near critical points. Journal of Computational Physics, 207, 2, 542-567, DOI: 10.1016/j.jcp.2005.01.023.

[23] Roy, C.J. (2005). Review of code and solution verification procedures for computational simulation. Journal of Computational Physics, 205, 1, 131-156, DOI: 10.1016/j.jcp.2004.10.036. 